\title{
HOLOMORPHIC FOLIATIONS IN RULED SURFACES
}

\author{
XAVIER GÓMEZ-MONT
}

\begin{abstract}
We analyse the universal families of holomorphic foliations with singularities in a ruled surface. In terms of Chern classes we determine the general and the special families. We also classify all nonsingular foliations.
\end{abstract}

A holomorphic foliation (with singularities) by curves in a complex manifold $M$ may be defined locally as the integral curves of a nonidentically zero holomorphic vector field and globally by a holomorphic bundle map from a line bundle on $M$ to the tangent bundle of $M, X: L \rightarrow T M$, uniquely defined up to multiplication by a never-vanishing holomorphic function. The set of all holomorphic foliations by curves in a compact manifold $M$ has a natural structure of a complex analytic variety, usually with infinitely many connected components, and in case $M$ is a Hodge manifold, then each connected component is compact [5]. The objective of this paper is to describe these components for the case of a ruled surface $S$. We organise the components $\mathscr{D}_{\alpha}$ according to the Chern classes of the defining bundle $L$, and we determine those elements in $H^{2}(S, Z)$ that appear as Chern classes of $L$. Using the notion of an ample direction $\beta$ (or of the ample cone) in $H^{2}(S, Z$ ), we observe a generic behaviour of the components $\mathscr{D}_{\alpha-m \beta}$ for $m$ large enough, and a special behaviour for $m$ small. The pattern observed resembles the pattern obtained in the theory of special divisors on a Riemann surface [7]. We also determine all nonsingular foliations in ruled surfaces and give topological and analytic descriptions of them. We finish by determining which foliations in ruled surfaces accept a change of complex structure on the leaves keeping the transversal structure fixed [6].

The paper is organised as follows. In $\S 1$ we describe the results of ruled surfaces (i.e. holomorphic $\mathbf{C} P^{1}$-bundles over a Riemann surface $B$ ) that we will need. In $\S 2$ we will obtain restrictions on the Chern classes $c(L) \in H^{2}(S, Z)$ of holomorphic foliations $X: L \rightarrow T S$ and describe the nonsingular foliations if the genus $g$ of $B$ is distinct from 1 . In $\S 3$ we analyse the case $g=1$. In $\S 4$ we describe the components $\mathscr{D}_{\alpha}$, as well as the subvariety of $\mathscr{D}_{\alpha}$ that parametrizes foliations that have an algebraic leaf of fixed fundamental class. Finally in $\S 5$ we apply the variational method of [6] to the ruled surface case.

Received by the editors October 15, 1987.

1980 Mathematics Subject Classification (1985 Revision). Primary 58F18, $14 \mathrm{~J} 26$.

Supported by Conacyt and University of Warwick. 
For an outline of the program of moduli spaces for foliations, one may consult [13], and [6] for a description of the tangent space to the moduli space.

\section{RULED SURFACES}

In this section we describe the results of ruled surfaces that will be needed in the following sections.

Definition 1.1. A ruled surface is a connected compact complex surface with a holomorphic map $\psi: S \rightarrow B$ to a nonsingular complex curve $B$ giving $S$ the structure of a holomorphic $\mathbf{C} P^{1}$-bundle over $B$.

The map $\psi$ induces on the level of cohomology an isomorphism $\psi^{*}: H^{1}$ $(B, Z) \simeq Z^{2 g} \rightarrow H^{1}(S, Z)$, where $g$ is the genus of $B$, and an injection $\psi^{*}: H^{2}(B, Z) \simeq Z \rightarrow H^{2}(S, Z)$ sending the fundamental class of $B$ to the Poincaré dual of a fiber of the ruling $\psi, f=\left[\psi^{-1}(b)\right]^{*}$. If $\sigma: B \rightarrow S$ denotes a holomorphic section of $\psi$ and $f^{\prime}$ denotes the Poincaré dual of $\sigma(B)$, then $f$ and $f^{\prime}$ form a basis of $H^{2}(S, Z)$ satisfying $f \cdot f=0$ and $f \cdot f^{\prime}=1$. We shall carry out computations in $H^{2}(S, Z)$ by expanding its elements in terms of $f$ and $h=f^{\prime}-\frac{1}{2}\left(f^{\prime} \cdot f^{\prime}\right) f$, using that $f \cdot h=1$ and $h \cdot h=0$.

Recall that the cup product in $H^{2}(S, Z)$ satisfies $\left[D_{1}\right]^{*} \cdot\left[D_{2}\right]^{*} \geq 0$ for any pair of distinct irreducible complex curves, and that it is strictly positive if $D_{1} \cap D_{2} \neq \varnothing$. Let $h+m f$ and $h+n f$ be the Poincaré duals of two distinct sections of $\psi$, then $(h+m f) \cdot(h+n f)=m+n \geq 0$, so they cannot be simultaneously negative. Let $e$ be the invariant of the ruled surface $\psi$ defined by

$$
e=-\min \left\{(m f+h)^{2} / m f+h=[\sigma(B)]^{*}, \sigma \text { section of } \psi\right\}
$$

and let $B_{0}=\sigma_{0}(B)$ be the image of a section attaining this minimum. The invariant $e$ satisfies $e \geq-g[8]$ and $\left[B_{0}\right]^{*}=-e f / 2+h$.

Lemma 1.2. Let $\psi: S \rightarrow B$ be a ruled surface, $C_{i}$ irreducible curves in $S$ distinct from $B_{0}$ and $D=\sum n_{i} C_{i}$ with $n_{i}$ positive integers, then $[D]^{*} \cdot f \geq 0$ and $[D]^{*} \cdot\left[B_{0}\right]^{*} \geq 0$ for $e \geq 0$ and $[D]^{*} \cdot h \geq 0$ for $e<0$. $D$ is an ample divisor if and only if we have strict inequalities above.

For the proof see [8, p. 382]. Let Amp $\subset \overline{\operatorname{Amp}} \subset H^{2}(S, Z)$ be the cohomology classes defined by the above inequalities (strict inequalities for Amp), or equivalently, if $[D]^{*}=a f+b h \in$ Amp then $b>0$ and $a>\frac{1}{2} b e$ for $e \geq 0$ and $a>0$ for $e<0$. For any positive divisor we have $[D]^{*} \in$ $\bigcup_{n \geq 0}\left(n\left[B_{0}\right]^{*}+\overline{\mathrm{Amp}}\right) \doteq \mathrm{Div}^{+}$. See Figure 1 . We will denote by $v+\mathrm{Div}^{+}$the region obtained by translating the region $\operatorname{Div}^{+}$by some element $v \in H^{2}(S, Z)$ and $-\operatorname{Div}^{+}=\left\{v \in H^{2}(S, Z) /-v \in \operatorname{Div}^{+}\right\}$, etc.

Topologically, the holomorphic line bundles are completely determined by their Chern class in $H^{2}(S, Z)$, and the holomorphic structures that one can put in each topological line bundle form a compact group variety of dimension 

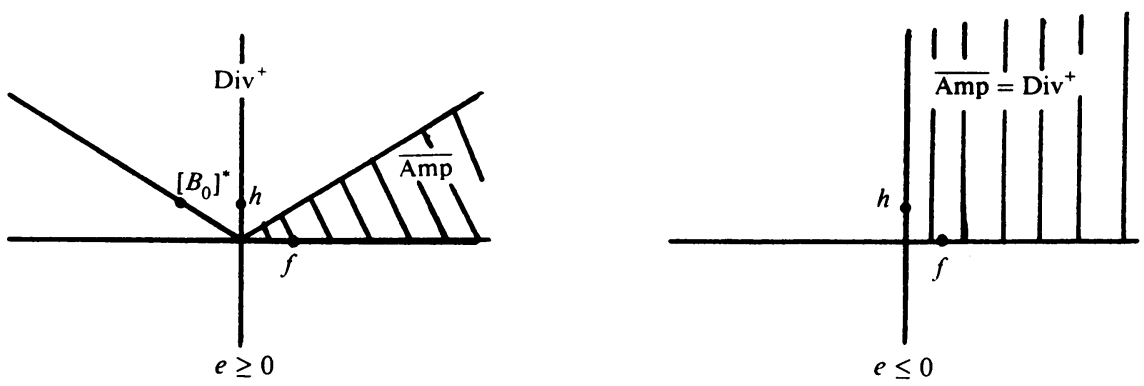

FIGURE 1

the genus $g$ of the base curve. This last assertion is obtained from the long exact sequence of cohomology of the exponential sheaf sequence [7] giving (1.1)

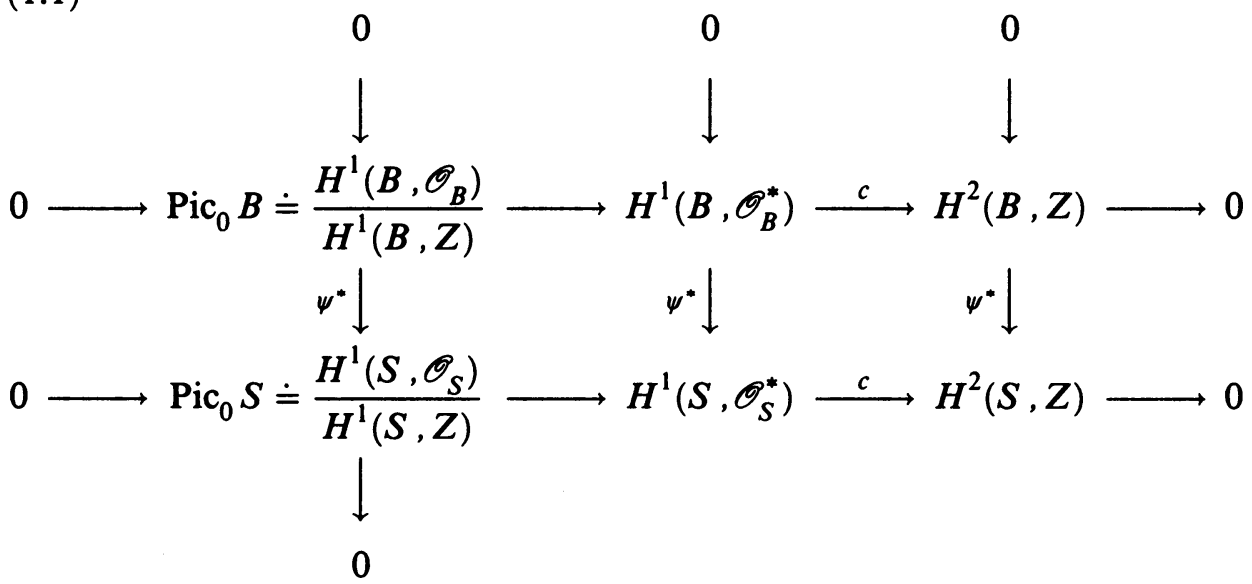

Lemma 1.3. The Chern class of the canonical bundle $\kappa_{S}=\Omega_{S}^{2}=\Lambda^{2} \Omega_{S}^{1}$ is $(2 g-2) f-2 h$ and we have for a holomorphic line bundle $L$ on $S$ :

(1) $H^{0}\left(S, \mathscr{O}_{S}(L)\right)=0$ if $c(L) \notin \mathrm{Div}^{+}$,

(2) $H^{2}\left(S, \mathscr{O}_{S}(L)\right)=0$ if $c(L) \notin c(\kappa)-\mathrm{Div}^{+}$,

(3) $H^{q}\left(S, \mathscr{O}_{S}(L)\right)=0$ for $q>0$ if $c(L) \in c(\kappa)+$ Amp,

(4) $H^{q}\left(S, \mathscr{O}_{S}(L)\right)=0$ for $q<2$ if $c(L) \in-$ Amp,

(5) If $c(L)=a f+b h$ then

$$
\chi(L) \doteq \sum_{i=0}^{2}(-1)^{i} \operatorname{dim} H^{i}\left(S, \mathscr{O}_{S}(L)\right)=(a+1-g)(b+1) .
$$

Proof. For the expression of $\kappa$ see [8, p. 373]. (1) is direct from Lemma 1.2 , as is (2) using Serre duality; (3) and (4) are direct from the KodairaNakano vanishing theorem [7] and Lemma 1.2. For (5), using the Riemann- 
Roch theorem [7],

$$
\begin{aligned}
\chi(L) & =\chi\left(1_{S}\right)+\frac{1}{2} c(L) \cdot(c(L)-c(\kappa)) \\
& =(1-g)+\frac{1}{2}(2 a b+2 a+2 b(1-g)) \\
& =a(b+1)+(b+1)(1-g)=(a+1-g)(b+1) .
\end{aligned}
$$

Let $T S$ be the tangent bundle of $S$ and let $\tau \hookrightarrow T S$ be the subline bundle defined as the kernel of the Jacobian of $\psi$,

$$
0 \rightarrow \tau \rightarrow T S \stackrel{D \psi}{\longrightarrow} \pi^{*} T B=N \rightarrow 0
$$

where $N$ is the normal bundle to the ruling.

Lemma 1.4. The Chern classes of $\tau$ and $N$ are

$$
c(\tau)=2 h, \quad c(N)=2(1-g) f .
$$

Proof. Since $c(T B)=2-2 g$ we have $c(N)=2(1-g) f . \kappa=\Lambda^{2} \Omega_{S}^{1} \cong \tau^{*} \otimes N^{*}$ has Chern class $(2 g-2) f-2 h$, so we have $c(\tau)=c\left(N^{*}\right)-c(\kappa)=2 h$.

\section{Foliations IN RULED SURFACES}

In this section we introduce foliations by curves in ruled surfaces and determine restrictions on the Chern classes of the line bundles defining them. Finally we determine all nonsingular foliations in ruled surfaces with $g \neq 1$.

A foliation by curves in the complex surface $S$ may be defined by nonidentically zero holomorphic vector fields $X_{i}$ defined on a covering $\left\{U_{i}\right\}$ of $S$, such that in $U_{i} \cap U_{j}$ we have $X_{i}=\xi_{i j} \cdot X_{j}$, with $\xi_{i j}$ never vanishing holomorphic functions. If $L$ denotes the holomorphic line bundle constructed with the cocycle $\left(\xi_{i j}^{-1}\right)$, then the $X_{i}$ give rise to a global map $X: L \rightarrow T S$. Conversely, such a map $X$ gives rise to a foliation by curves, and two different maps $X$ and $X^{\prime}$ give rise to the same foliation if and only if $X^{\prime}=f X$ with $f$ a never-vanishing function on $S$ (see [5]).

Definition 2.1. A foliation by curves in the connected complex surface $S$ is a nonidentically zero holomorphic bundle map $X: L \rightarrow T S$ from the line bundle $L$ to the tangent bundle of $S$.

We will obtain restrictions on the Chern classes of the line bundle $L$ of a foliation in a ruled surface $S$ given by $X: L \rightarrow T S$. Composing with (1.2) we obtain a commutative diagram

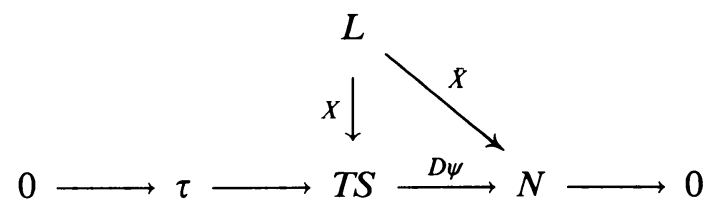



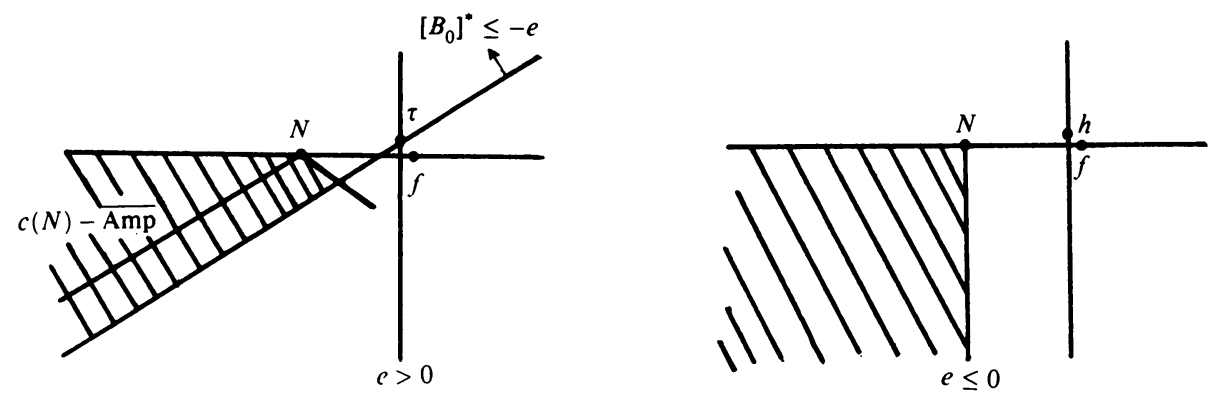

FIGURE 2

Proposition 2.2. Let $X: L \rightarrow T S$ be a bundle map defining a foliation by curves on the ruled surface $\psi: S \rightarrow B$ and assume that $X$ vanishes at most on a discrete set of points, then either

(1) $X(L)=\tau$, or

(2) $L$ is biholomorphic to $N$, or

(3) $c(L) \in C(N)-\overline{\mathrm{Amp}}$, or

(4) $e>0$ and $c(L) \in c(N)-\mathrm{Div}^{+}$and $c(L) \cdot\left[B_{0}\right]^{*} \leq-e$.

Proof. If $X \equiv 0$, then $X$ factors to $\tau, X: L \rightarrow \tau$, and since $X$ vanishes at most on a discrete set of points by hypothesis then it is nonvanishing, since maps between line bundles vanish on a curve or not at all. Hence $X(L)=\tau$. If $\bar{X}$ never vanishes, then $X$ gives an isomorphism from $L$ to $N$, so $X$ satisfies 2 . Assume now that $\bar{X}$ has a nontrivial vanishing set $m B_{0}+D$, with $D \in \overline{\mathrm{Amp}}$. Since $X$ is a global section of $L^{*} \otimes N$, we have $c(L)=c(N)-\left[m B_{0}+D\right]^{*}$. If $e \leq 0$ then $c\left(B_{0}\right) \in \overline{\mathrm{Amp}}$, so that independently of the value of $m$ we have $c(L) \in c(N)-\overline{\text { Amp }}$. If $e>0$ and $m=0$, then again $c(L) \in c(N)-\overline{\mathrm{Amp}}$, so assume $e>0$ and $m>0$. The fact that $X$ vanishes on $B_{0}$ means that $X$ restricted to $B_{0}$ takes values in $\tau$, but then $X$ restricted to $B_{0}$ must vanish as many times as the Chern class of $\left.L^{*} \otimes \tau\right|_{B_{0}}$ which is

$$
0 \leq(-c(L)+2 h) \cdot\left[B_{0}\right]^{*}=-c(L) \cdot\left[B_{0}\right]^{*}-e
$$

so that $c(L) \cdot\left[B_{0}\right]^{*} \leq-e$. See Figure 2 .

Remark. Note that condition 4 is not contained in 3 only if $g>1$ and $0<$ $e<2 g-2$.

Any foliation by curves in $S$ may be obtained by multiplying a map $X: L \rightarrow$ $T S$ vanishing at most on a discrete set of points with a section $g$ of a line bundle $L_{1}$ vanishing on a curve in $S, g X: L \otimes L_{1}^{*} \rightarrow T S$. Combining Lemma 1.2 and Proposition 2.2 we obtain restrictions on the possible Chern classes of the tangent fields to foliations in $S$.

Corollary 2.3 (Rigidity of the ruling). If $X: L \rightarrow T S$ is a foliation by curves with $c(L)=c(\tau)$, where $\tau \hookrightarrow T S$ is the tangent field to the ruling, then $L$ is biholomorphic to $\tau$ and $X(L)=\tau$. 
Proof. Let $C$ be the codimension 1 subvariety where $X$ vanishes, suppose it is defined as the zero set of $g \in H^{0}\left(S, L_{1}\right)$ and let $Y: L \otimes L_{1} \rightarrow T S$ be the foliation defined by $Y=g^{-1} X$, vanishing on a discrete set of points. The Chern class of $L \otimes L_{1}$ lies in $c(\tau)+\mathrm{Div}^{+}$, so using Proposition 2.2 we see that the only possibility is $Y\left(L \otimes L_{1}\right)=\tau$ so that $c\left(L_{1}\right)=0$, and hence $L_{1}$ is the trivial line bundle and $C=\varnothing$. Again by Proposition 2.2, $X(L)=\tau$, which proves the corollary.

Proposition 2.4. Let $X: L \rightarrow T S$ be a foliation by curves in $S$ vanishing at most on a discrete set of points and let $a f+b h$ be the Chern class of $L$, then

(1) Counting multiplicities, $X$ vanishes on $2(a+g-1)(b-1)+2(1-g)$ points.

(2) If all the zeros of $X$ vanish with multiplicity one, then

$$
\sum_{X(p)=0} \frac{\operatorname{Tr}^{2} D X(p)}{\operatorname{Det} D X(p)}=2(2-2 g-a)(2-b) .
$$

Proof. The Chern classes $c_{1}$ and $c_{2}$ of $S$ are

$$
\begin{aligned}
1+c_{1} t+c_{2} t^{2} & =\left(1+c_{1}(\tau) t\right) \cdot\left(1+c_{1}(N) t\right)=(1+2 h t)(1+(2-2 g) f t) \\
& =1+(2 h+(2-2 g) f) t+4(1-g) t^{2} .
\end{aligned}
$$

The inverse of the total Chern class of $L$ is $1-(a f+b h) t+2 a b t^{2}$, and the Chern class of the virtual bundle $T S-L$ (or equivalently of the sheaf $\Theta_{S} \mid X \mathscr{O}_{S}(L)$ ) is

$$
\begin{aligned}
& \left(1+(2 h+(2-2 g) f) t+4(1-g) t^{2}\right)\left(1-(a f+b h) t+2 a b t^{2}\right) \\
& \quad=1+((2-b) h+(2-2 g-a) f) t+(2 a b-2 a-(2-2 g) b+4(1-g)) t^{2}
\end{aligned}
$$

so that

$$
\begin{gathered}
c_{1}(T S-L)^{2}=2(2-b)(2-2 g-a), \\
c_{2}(T S-L)=2 a(b-1)-2(1-g)(b-1)+2(1-g) \\
=2(a+g-1)(b-1)+2(1-g) .
\end{gathered}
$$

Viewing $X$ as a section of the $C^{2}$-fiber bundle $T S \otimes L^{*}$ and $X(S)$ as a subvariety of $T S \otimes L^{*}$, then the intersection number of $X(S)$ with the zero subvariety $0_{T S \otimes L^{*}}$ of $T S \otimes L^{*}$ is the number of points where $X$ vanishes, counting multiplicities. These two subvarieties of $T S \otimes L^{*}$ are homologous, and their normal bundles are isomorphic to the restriction of $T S \otimes L^{*}$; hence by $[9, p .72]$ we have

$$
\sum_{X(p)=0} \mu(X, p)=X(S) \cdot 0_{T S \otimes L^{*}}=c_{2}\left(T S \otimes L^{*}\right)[S] .
$$

This proves part 1. Chern in [4] proves this formula for points with multiplicity one as a generalization of the Poincaré-Hopf index formula, where he also 
proves

$$
\sum_{X(p)=0} \frac{\operatorname{tr}^{2} D X(p)}{\operatorname{det} D X(p)}=c_{1}\left(T S \otimes L^{*}\right)^{2}[S] .
$$

These formulas, together with the above computations of the Chern classes of $T S \otimes L^{*}$, prove the Proposition.

If we plot in the $(a, b)$-plane the curves defined by

$$
2(a+g-1)(b-1)+2(1-g)=n, \quad n \geq 0,
$$

we obtain a family of hyperbolas whose asymptotes are $a=1-g$ and $b=$ 1 , and each foliation with isolated singularities whose Chern class lies on the hyperbola has $n$ singular points.

Definition 2.5. We say that a foliation $X: L \rightarrow T S$ in the ruled surface $\psi: S \rightarrow$ $B$ is a Riccati foliation if the Chern class of $L$ lies in $\psi^{*} H^{2}(B, Z)$ (or equivalently, if the restriction of $L$ to any fiber of $\psi$ is topologically trivial, or equivalently, if $L$ is the pull back of a holomorphic bundle in $B$ ).

Proposition 2.6. Let $X: L \rightarrow T S$ be a Riccati foliation with $c(L)=a f$, then

(1) Counting multiplicities there are $2-2 g-a \geq 0$ fibers of the ruling that are leaves of the foliation (after removing the singular points on them), and all the rest of the leaves of the foliation intersect transversely the remaining fibers of the ruling.

(2) After removing the fibers that are leaves of $X$, the foliation is constructible by suspending a representation $\rho: \pi_{1}\left(B-\left\{b_{1}, \ldots, b_{r}\right\}\right) \rightarrow \operatorname{PSL}(2, \mathbf{C})$.

Proof. (1) Let $X$ be as in (2.1), viewed as a section of $L^{*} \otimes N$. This bundle has Chern class $(2-2 g-a) f$. The divisor $X=0$ consists of a finite union of fibers of $\psi$, since $(2-2 g-a) f \cdot f=0$ and for any curve $C$ not a fiber we have $f \cdot[C]^{*}>0$. The set of points where $\bar{X}$ vanishes is the set of points where the foliation and the ruling are tangent, hence the fibers $\bar{X}=0$ are leaves of the foliation. Counting multiplicities, there are $2-2 g-a$ such fibers. Outside these fibers $X$ does not vanish, so the foliation is transverse to the ruling.

(2) Painleve's theorem [12, p. 34] shows that choosing any path in $B-\psi(\bar{X}=$ $0)$, the process of lifting the path within a leaf gives a biholomorphism from the initial fibre to the final fibre. This shows the uniform covering property of the foliation and gives a monodromy map $\rho$ as stated. The foliation in $S-(\bar{X}=0)$ is biholomorphic to the suspension of $\rho$. This finishes the proof.

Remarks. (1) Observe that the Chern class of Riccati foliations lie on the $f$ axis in Figure 2, and hence they lie on one of the lines bounding the region of admissible possibilities described by Proposition 2.2.

(2) Given a representation $\rho: \pi_{1}\left(B-\left\{b_{1}, \ldots, b_{r}\right\}\right) \rightarrow \operatorname{PSL}(2, \mathrm{C})$, by suspending it we obtain a nonsingular holomorphic foliation in the ruled surface $\psi^{\prime}: S^{\prime} \rightarrow B-\left\{b_{1}, \ldots, b_{r}\right\}$. If $D_{i}$ is a disc in $B$ surrounding the puncture $b_{i}$, then the foliation over $D_{i}-\left\{b_{i}\right\}$ is determined by $\rho\left(\partial D_{i}\right) \in \operatorname{PSL}(2, \mathrm{C})$. We 
may construct on $D_{i} \times \mathbf{C} P^{1}$ a singular foliation with expression in $D_{i} \times \mathbf{C}$ of the form $z(\partial / \partial z)+\lambda_{i} w(\partial / \partial w)$ or $z(\partial / \partial z)+\partial / \partial w$, having monodromy over $D_{i}$ a transformation biholomorphic to $\rho\left(\partial D_{i}\right)$. Since over $D_{i}-\left\{b_{i}\right\}$ both foliations are biholomorphic, we may glue them by means of these biholomorphisms to obtain a Riccati foliation on $\psi: S \rightarrow B$. It is a delicate problem to determine which ruled surface $S$ one obtains from the representation $\rho$ and the scalars $\lambda_{i}$. This construction has its origins in Birkoff's solution to the Riemann problem [2], and has been recently considered by Martinet-Ramis [8] and by Alcides Lins. One may use other models in $D_{i} \times \mathbf{C} P^{1}$ to compactify $S^{\prime}$.

Proposition 2.7. Let $\psi: S \rightarrow B$ be a ruled surface with $g \neq 1$. A nonsingular foliation by curves $X: L \rightarrow T S$ in $S$ distinct from the ruling is everywhere transversal to the ruling and the foliation is constructible by suspending a representation $\rho: \pi_{1}(B) \rightarrow P S L(2, \mathbf{C})$.

Proof. The only elements of $H^{2}(S, Z)$ that satisfy the two equations of Proposition 2.4 equated to zero are $2 h$ and $2(1-g) f$. By Corollary 2.3 then $c(L)$ is $2(1-g) f$. If $\bar{X}: L \rightarrow N$ is as in $(2.1)$, then $\bar{X}$ is a section of $L^{*} \otimes N$, which has Chern class zero; so $\bar{X}=0$ if $L \neq N$ and $\bar{X}$ is constant if $L=N$. If $L \neq N$, then $X$ factors to an isomorphism $X: L \rightarrow \tau$, which is absurd since they have distinct Chern classes, so $L=N$. By the same reason as above, $\bar{X}: L \rightarrow N$ is a nonvanishing constant, so $X$ is everywhere transversal to the ruling. The last statement follows from Proposition 2.6, since $L=N=\psi_{1}^{*}(T B)$, so $X$ is a Riccati foliation.

\section{Elliptic RULED SURFACES}

A ruled surface is called elliptic if the genus of the base curve is 1 . The objective of this section is to show that some elliptic surfaces admit nonsingular foliations distinct from the ruling or nonsingular Riccati foliations. We will describe which surfaces admit them, and we will give analytic and topological description of the foliations.

Proposition 2.4 implies that if $X: L \rightarrow T S$ is a nonsingular foliation by curves in the elliptic ruled surface $\psi: S \rightarrow E$, then the Chern class of $L$ is of the form $b h$. If $b>0$, then $X(L)=\tau$ by Proposition 2.2, and if $b=0$, then $X$ is a nonsingular Riccati foliation with $L$ the trivial line bundle. Using Proposition 2.2 we see that for $e>0$, there are no other nonsingular foliations. Hence we are reduced to analyse elliptic ruled surfaces with $e=0,-1$ and $c(L)=b h$ with $b<0$.

Example 3.1. Let $P(z) / Q(z) d z$ be a rational 1 -form on the Riemann sphere $\overline{\mathbf{C}}$, and consider the nonsingular foliation in $\overline{\mathbf{C}} \times \mathbf{C}$, with affine coordinates $(z, w)$ defined by $P(z) d z-Q(z) d w$. Outside of the zeros of $Q$, the leaves of the foliation are the graphs of the multiple valued function

$$
w=\int \frac{P(z)}{Q(z)} d z
$$


From the above expression, it is clear that the foliation is invariant under translations in the $w$-coordinate and has nontrivial monodromy around the zeros of $Q$ if the integrand has nonintegral residue. Let $\lambda$ be a nonreal complex number and let $E$ be the elliptic curve obtained by moding out $\mathbf{C}$ by the group generated by 1 and $\lambda$. The above foliation descends to a nonsingular foliation in $\bar{C} \times E$.

Proposition 3.2. For every nonpositive $b \leq 0$ there are nonsingular foliations with Chern class bh in the elliptic ruled surface $\psi: \overline{\mathbf{C}} \times E \rightarrow E$.

Proof. In the proof, we will actually give a description of these foliations: If $\pi: \overline{\mathbf{C}} \times E \rightarrow \overline{\mathbf{C}}$ denotes the projection to the first factor and if the foliation is not defined by $d \pi=0$, then it has $-b+2 \pi$-fibers as leaves and the remaining leaves of the foliation are transverse to the $\pi$-fibers, giving rise as in the Riccati foliations to a holonomy map $\pi_{1}\left(\overline{\mathbf{C}}-\left\{t_{1}, \ldots, t_{-b+2}\right\}\right) \rightarrow \operatorname{Aut}_{0}(E)=E$. Let $\widetilde{E} \simeq \mathbf{C}$ be the universal covering space of $E$, and $(z, \omega)$ affine coordinates of $\overline{\mathbf{C}} \times \mathbf{C}$; we will now show that these foliations are described by 1 -forms as in Example 3.1 with $P$ and $Q$ of degree at most $-b$ and $-b+2$ respectively, without common factors and at least one of them attains the top degree.

Denote by $H^{d}$ the line bundle on $S=\overline{\mathbf{C}} \times E$ obtained by pulling back the line bundle on $\overrightarrow{\mathbf{C}}$ with Chern class $d$. It follows from (1.1) that all line bundles on $S$ with Chern class $b h$ may be expressed in a unique way as $H^{D} \otimes \eta$, with $\eta$ a line bundle on $S$ with Chern class $0, \eta \in \operatorname{Pic}_{0}(S)=\psi^{*} \operatorname{Pic}_{0}(E) \simeq E$.

The tangent bundle of $S$ decomposes as a direct sum and the foliation may be expressed as

$$
X=\left(X_{1}, X_{2}\right): H^{b} \otimes \eta \rightarrow \pi^{*} T \overline{\mathbf{C}} \oplus \psi^{*} T E=H^{2} \oplus 1_{S}
$$

Restricting $X$ to a set of the form $E \times t$, the line bundles in (3.2) simplify to give $\left.X\right|_{E \times t}:\left.\eta\right|_{E \times t} \rightarrow 1_{E \times t} \oplus 1_{E \times t} \cdot \eta$ has Chern class 0 , but there are no nontrivial maps on $E$ from $\eta$ to $1_{E}$ unless $\eta=1_{E}$ and then the only maps are constants. Hence we deduce that $\eta=\psi^{*}(\eta)$ is the trivial line bundle.

We may view $X_{1}$ as a global section of the bundle $\pi^{*} T \overline{\mathbf{C}} \otimes H^{-b}$. Note that this bundle restricted to sets of the form $E \times t$ is trivial, hence $X_{1}$ is constant along sets of the form $E \times t$ and it has to vanish on $-b+2$ subsets of this form, if $X_{1} \neq \equiv 0$. But if $X_{1}$ vanishes on $E \times t_{i}$, then $E \times t_{i}$ is a leaf of the foliation. Hence the foliation has $-b+2 \pi$-vertical fibers, and it is transverse to the remaining $\pi$-fibers. Now we are exactly in the same situation as in the Riccati foliations. Dualising (3.2), we obtain

$$
0 \rightarrow H^{b-2} \stackrel{\varphi}{\longrightarrow} \pi^{*} \Omega_{C}^{1} \oplus \psi^{*} \Omega_{E}^{1}=H^{-2} \oplus 1_{S} \rightarrow H^{-b} \rightarrow 0
$$

so that $\varphi$ may be viewed as a global section of $H^{-b} \oplus H^{-b+2}$, so $\varphi=P(z) d z+$ $Q(z) d w$ with $P$ and $Q$ polynomials of degree at most $-b$ and $-b+2$. This finishes the proof of the proposition.

The rest of this section will be devoted to prove the following theorem. 
Theorem 3.3. Nonsingular foliations distinct from the ruling or nonsingular Riccati foliations only appear on elliptic ruled surfaces that are covered in an unbranched manner by the product of an elliptic curve with $\overline{\mathbf{C}}$.

Pulling back the new nonsingular foliations to $\overline{\mathbf{C}} \times E$, the proof of Proposition 3.2 shows that the foliation may be described by a 1 -form as in (3.1).

We will give the proof in several steps. We will begin by analysing ruled surfaces $\psi: S \rightarrow E$ with $e=0$. It is known that $S$ may be obtained by projectivising a holomorphic vector bundle $G$ of rank two on $E$ (see [8]), and it follows from Atiyah's classification of vector bundles over an elliptic curve [1] that we may take $G$ to be:

(a) $G=1_{E} \oplus \eta$, a direct sum of the trivial line bundle on $E$ with a holomorphic line bundle $\eta$ with Chern class $0, \eta \in \operatorname{Pic}_{0}(E)$. The representation of $S$ in this form is unique except that $\eta$ and its inverse $\eta^{*}$ give rise to the same surface.

(b) $G$ is the only nonsplit extension of the trivial line bundle by the trivial line bundle on $E$

$$
0 \rightarrow 1_{E} \rightarrow G \rightarrow 1_{E} \rightarrow 0 .
$$

To simplify notation, an upper index $d$ over a line bundle means tensor product of the line bundle $d$ times. The construction of the complex surface $S=\operatorname{Proj}(G)$ also comes provided with a line bundle $H$ over $S$ that restricts to every fibre $\psi^{-1}(b) \simeq \overline{\mathbf{C}}$ as the hyperplane bundle over $\overline{\mathbf{C}}$. It is also known that for $d>0 R^{q} \psi_{*}\left(\mathscr{O}_{S}\left(H^{d}\right)\right)=0$ for $q>0$ and $\psi_{*}\left(\mathscr{O}_{S}\left(H^{d}\right)\right)$ is isomorphic to the sheaf of holomorphic sections of the $d$ th symmetric power of $G$. Note that for case (a) the $d$ th symmetric product of $G$ is $1_{E} \oplus \eta \oplus \cdots \oplus \eta^{d}$. One also knows that the Chern class of $H$ is $1 \cdot h[8$, p. 371].

One may generalize the Euler sequence to obtain the relative Euler sequence over $\operatorname{Proj}(G)$ (see $[8$, p. 253]):

$$
0 \rightarrow 1_{S} \rightarrow G^{*} \otimes H_{S} \rightarrow \tau \rightarrow 0
$$

where $\tau$ is the line bundle of tangent vectors to the fibers of $\psi$ as in (1.2). One deduces from (3.4) that $\tau$ is isomorphic to the determinant bundle of $G^{*} \otimes H_{S}$, that is equal to $\eta^{*} \otimes H_{S}^{2}$ in case (a) and to $H_{S}^{2}$ in case (b).

We will identify the topologically trivial bundles on $S$ and on $E$ by means of $\psi^{*}$ in (1.1). A point $\xi \in \operatorname{Pic}_{0}(E)$ is a torsion point if for some positive integer $n$ we have $\xi^{n}=1_{E}$, and the smallest such $n$ is the order of $\xi$.

Lemma 3.4. Let $\eta$ be a nontorsion point of $\operatorname{Pic}_{0}(E)$ and $\psi: S=\operatorname{Proj}\left(1_{E} \oplus \eta\right) \rightarrow$ $E$ the ruled surface over $E$ obtained by projectivising the vector bundle $1_{E} \oplus \eta$. Then

(1) For every $c>0$ and every $\xi \in \operatorname{Pic}_{0}(S)$ we have

$$
H^{0}\left(S, \mathscr{O}_{S}\left(H^{c} \otimes \xi\right)\right) \simeq \begin{cases}0 & \text { if } \xi \neq 1_{S}, \eta^{-1}, \ldots, \eta^{-c} \\ \mathbf{C} & \text { if } \xi=1_{S}, \eta^{-1}, \ldots, \eta^{c}\end{cases}
$$


(2) The nonsingular Riccati foliations in $S$ form a 1-dimensional family.

(3) There are no foliations in $S$ with Chern class $b h, b<0$.

Proof. (1) Using the higher direct image sheaves we have

$$
H^{0}\left(S, \mathscr{O}_{S}\left(H^{c} \otimes \xi\right)\right)=H^{0}\left(E, \mathscr{O}_{E}\left(1 \oplus \cdots \oplus \eta^{c}\right) \otimes \xi\right) .
$$

Recall that $\eta^{b} \otimes \xi$ has Chern class zero, so $H^{0}\left(E, \mathscr{O}_{E}\left(\eta^{b} \otimes \xi\right)\right)$ is 0 unless $\eta^{b} \otimes \xi=1_{E}$. By the assumption that $\eta$ is a nontorsion point this happens at most once for $\xi=\eta^{-b}$. Let $f_{i} \in H^{0}\left(S, \mathscr{O}_{S}\left(H \otimes\left(\eta^{*}\right)^{i}\right), i=0,1\right.$, be a basis. Then clearly $f_{0}^{c-d} \cdot f_{1}^{d}$ is a basis of the 1-dimensional vector space $H^{0}\left(S, \mathscr{O}_{S}\left(H^{c} \otimes\left(\eta^{*}\right)^{d}\right)\right), d \leq c$.

(2) The long exact sequence of higher direct image sheaves of (1.2) gives the short exact sequence of sheaves on $E$

$$
0 \rightarrow \mathscr{O}_{E}\left(\eta^{-1} \otimes\left(1_{E} \oplus \eta \oplus \eta^{2}\right)\right) \rightarrow \psi_{*} \mathscr{O}_{S}(T S) \rightarrow \mathscr{O}_{E} \rightarrow 0
$$

where we have used that $\tau=\eta \otimes H^{2}$ and $N=1_{S}$. The long exact sequence of cohomology gives

$$
0 \rightarrow \mathbf{C} \rightarrow H^{0}\left(S, \mathscr{O}_{S}(T S)\right) \rightarrow \mathbf{C} \stackrel{\delta}{\longrightarrow} \mathbf{C} \rightarrow H^{1}\left(S, \mathscr{O}_{S}(T S)\right) \rightarrow \mathbf{C} \rightarrow 0 .
$$

From deformation theory we know that the dimension of $H^{1}\left(S, \mathscr{O}_{S}(T S)\right)$ is at least two, since we know an effective family of deformations of $S$ on two parameters (vary $E$ and $\eta$ ), so it has dimension $2, \delta=0$ and $H^{0}\left(S, \mathscr{O}_{S}(T S)\right.$ ) has dimension 2 . The nonsingular Riccati foliations are described by the holomorphic vector fields since $N=1_{S}$. If $X$ is a vector field tangent to $\tau$ and $Y$ is a vector field projecting to 1 in $N$, then the Riccati foliations are parametrized by $\alpha X+Y$. A topological description of these foliations is obtained by observing that the vector bundle $1 \oplus \eta$ is flat and that it may be constructed from a representation of $\pi_{1}(E)$ by sending the two generators to $\left(\begin{array}{ll}1 & 0 \\ 0 & a_{1}\end{array}\right)$ and $\left(\begin{array}{ll}1 & 0 \\ 0 & a_{2}\end{array}\right)$ with $a_{1} / a_{2}$ the invariant of the extension $\eta$. By suspending these representations we obtain the nonsingular Riccati foliations.

(3) For $b<0$ and $\xi \in \operatorname{Pic}_{0}(S)$, nonsingular foliations with tangent bundle $H^{b} \otimes \xi$ correspond to never-vanishing global sections of $T S \otimes H^{-b} \otimes \xi^{*}$. Tensoring (1.2) with $H^{-b} \otimes \xi^{*}$ gives

$$
0 \rightarrow \eta^{*} \otimes H^{-b+2} \otimes \xi^{*} \rightarrow T S \otimes H^{-b} \otimes \xi^{*} \rightarrow H^{-b} \otimes \xi^{*} \rightarrow 0 .
$$

Note that if a section of the middle term projects to 0 in the last, that foliation is then tangent to the ruling. So in order to have nontrivial foliations we need $H^{0}\left(S, \mathscr{O}_{S}\left(H^{-b} \otimes \xi^{*}\right)\right) \neq 0$ which by part (1) happens only if $\xi=\eta^{d}$ for $d=$ $0, \ldots,-b$, in which case it is 1-dimensional. Using the basis from parts 1 and 2 we can parametrize the sections of $T S \otimes H^{-b} \otimes \xi^{*}$ as $f_{0}^{-b-d} f_{1}^{d}(\alpha X+Y)$ and hence conclude that all its sections vanish on the curves defined by $f_{i}=0$, $i=0,1$. Hence there are no nonsingular foliations arising from $H^{b} \otimes \xi$. The lemma is then proved. 
Lemma 3.5. Let $\eta$ be a torsion point of order $n$ in $\operatorname{Pic}_{0}(E)$ and $S=\operatorname{Proj}(1 \oplus \eta)$, then there are nonsingular foliations in $S$ different from the ruling and Riccati foliations.

Proof. The transition coordinates of $\eta$ may be given by a multiplier $e^{2 \pi i \chi}$, where $\chi: \pi_{1}(E) \rightarrow Z / n Z$ is a group homomorphism. Let $E^{\prime}$ be the elliptic curve defined by $\operatorname{Ker} \chi$,

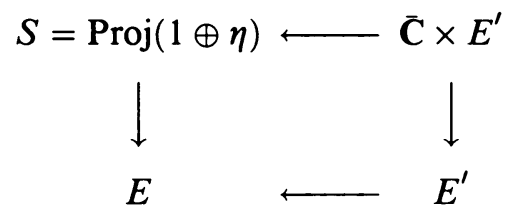

$S$ is pulled back to $E^{\prime}$ as $\overline{\boldsymbol{C}} \times E^{\prime}$, and a nonsingular foliation in $S$ pulls back to a foliation in $\overline{\mathbf{C}} \times E^{\prime}$ invariant under the covering group. The equation of the foliation in $\mathbf{C} \times \mathbf{C}$ is by Proposition 3.2 of the form

$$
\sum a_{j} z^{j} \frac{\partial}{\partial z}+\sum b_{j} z^{j} \frac{\partial}{\partial w}
$$

and it must be equivariant with respect to the covering group generated by

$$
T_{1}(z, w)=\left(e^{2 \pi i a / n} \cdot z, w+1\right), \quad T_{2}(z, w)=\left(e^{2 \pi i b / n} \cdot z, w+\lambda\right)
$$

where $a$ and $b$ are integers between 0 and $n-1$. This equivariance is reflected in (3.5) by sums of the form

$$
\sum_{j \equiv l+1(\bmod n)} a_{j} z^{j} \frac{\partial}{z_{j}} \sum_{j \equiv l(\bmod n)} b_{j} z^{j} \frac{\partial}{\partial w}
$$

where $l$ is an integer between 0 and $n-1$. Since we are interested in the nonsingular foliations we need $a_{0} \neq 0$ or $b_{0} \neq 0$, so that $l=0,-1$ and the tangent bundles to the foliation is $\overline{\mathbf{C}} \times E^{\prime}$ are isomorphic to $H^{m n}, H^{m n-1}$ or $H^{m n-2}$. This proves the lemma.

Lemma 3.6. Let $S=\operatorname{Proj}(G)$ where $G$ is the nonsplit extension (3.3). Then

(1) The nonsingular Riccati foliations in $S$ form a 1-dimensional family.

(2) There are no nonsingular foliations which are tangent to line bundles with Chern class bh, $b<0$.

Proof. $G$ may be constructed as $\mathbf{C} \times \mathbf{C}^{2}$ divided by the group generated by $(z ; s, t) \rightarrow(z+1 ; s, t),(z ; s, t) \rightarrow(z+\lambda, s+t, t)$ and similarly $S$, using homogeneous coordinates $(z ; s: t) \in \mathbf{C} \times \overline{\mathbf{C}}$. Taking affine coordinates $(z, s) \in$ $\mathbf{C} \times \mathbf{C} \subset \mathbf{C} \times \overline{\mathbf{C}}$ we have covering transformations

$$
(z, s) \rightarrow(z+1, s), \quad(z, s) \rightarrow(z+\lambda, s+1) .
$$

A holomorphic vector field on $S$ lifts to a holomorphic vector field on $\mathbf{C} \times \mathbf{C}$ invariant under (3.6) and holomorphic on $s=\infty$. Such a vector field must be of the form

$$
A(z) \frac{\partial}{\partial z}+\left(B_{2}(z) s^{2}+B_{1}(z) s+B_{0}(z)\right) \frac{\partial}{\partial s}
$$


in order that it be holomorphic at $s=\infty$. Invariance under (3.6) implies that $A$ is constant and $B_{i}(z+1)=B_{i}(z)$. The second condition of (3.6) implies first that $B_{2}(z+\lambda)=B_{2}(z)$, hence $B_{2}$ is constant and also $2 B_{2}+B_{1}(z+\lambda)=B_{1}(z)$. Hence $\partial_{z} B_{1}(z+\lambda)=\partial_{z} B_{1}(z)$ so that $B_{1}(z)=K z+L$. The condition $B_{1}(z)=$ $B_{1}(z+1)$ forces then $K=0$, so that $B_{1}(z)$ is constant, but then $B_{2}=0$. Arguing identically for $B_{0}$, we conclude that $B_{1}=0$ and $B_{0}$ is a constant. Hence we are reduced to vector fields of the form $A(\partial / \partial z)+B(\partial / \partial s)$. At $s=\infty$ it takes the form $A(\partial / \partial z)-B t^{2}(\partial / \partial t)$. Hence foliations arising from the normal bundle are parametrized by $\partial / \partial z+B(\partial / \partial s)$. This proves part 1 .

Let $X$ be the tangent map to a nonsingular foliation with Chern class $b h$, $b<0$. For some $\xi$ in $\operatorname{Pic}_{0}\left(S_{0}\right)$ it may be written as

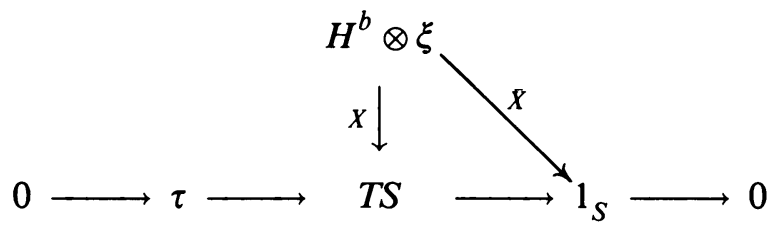

Let $E_{0}$ be the section of $S \rightarrow E$ defined by $t=0$ (or $s=\infty$ ). $H$ restricted to $E_{0}$ is holomorphically trivial, so that $\left.\bar{X}\right|_{E_{0}}:\left.\xi\right|_{E_{0}} \rightarrow 1_{S}$. If $\left.\bar{X}\right|_{E_{0}}$ is identically zero, then $X$ factors to an isomorphism $\left.X\right|_{E_{0}}:\left.\left.\xi\right|_{E_{0}} \rightarrow \tau\right|_{E_{0}}=\left.H^{2}\right|_{E_{0}}=1_{E_{0}}$ so that $\xi$ is trivial. If $\left.X\right|_{E_{0}}$ is not identically zero, then it is an isomorphism since $c(\xi)=0$; so $\xi=1_{S}$ for both cases.

Let $f$ be the section of $H$ that vanishes on $E_{0}$, and consider $Y=f^{b} X: 1 \rightarrow$ $T S$, as a rational vector field of $S$ with a pole of order $b$ along $E_{0}$. Writing $Y$ in coordinates $(z, s)$ we obtain

$$
Y=\sum_{i=0}^{m} A_{i}(z) s^{i} \frac{\partial}{\partial z}+\sum_{i=0}^{n} B_{i}(z) s^{i} \frac{\partial}{\partial s}
$$

where $m$ and $n$ are finite since $Y$ has a pole at $s=\infty$, and $A_{i}$ and $B_{i}$ are entire functions. Repeating the same argument as in part 1 we conclude that $Y=A(\partial / \partial z)+B(\partial / \partial s)$. Hence $X=f^{-b} Y$, with $Y$ holomorphic. Since $X$ should vanish on isolated points and $f$ vanishes on $E_{0}$, we conclude that there are no nonsingular foliations with Chern class $b h$ with $b<0$, and the lemma is proved.

Proof of Theorem 3.3. The preceding lemmas show that for $e \neq-1$ foliations in elliptic ruled surfaces are covered in an unbranched manner by foliations in $E^{\prime} \times \mathrm{C}$. We will show that this is also the case if $e=-1$.

Over an elliptic curve $E$ there is a unique ruled surface $S$ with $e=-1$ [8, p. 377]; and if $E=\mathbf{C} /((1 / 2) Z+(\lambda / 2) Z)$ and $E^{\prime}=\mathbf{C} /(Z+\lambda Z)$ then $S$ may be obtained from $\overline{\mathbf{C}} \times E^{\prime}$ by quotienting with the involutions $T_{1}(z, w)=$ $\left(-z, w+\frac{1}{2}\right), T_{2}(z, w)=(1 / z, w+\lambda / 2)$. (This may be seen by observing that $e \leq 0$ since it is covered by $\overline{\mathrm{C}} \times E^{\prime}$, and it has no sections $\sigma$ with $\left([\sigma(E)]^{*}\right)^{2}=0$ since $T_{1}$ and $T_{2}$ have different fixed points.) Nonsingular 
foliations in $S$ correspond to nonsingular foliations in $\overline{\mathrm{C}} \times E^{\prime}$ invariant under $T_{1}$ or $T_{2}$ or equivalently to $T_{1}$ and $T_{2}$ covariant maps $X: L \rightarrow T\left(\overline{\mathbf{C}} \times E^{\prime}\right)$. If $c(L)=-n h$ with $n \geq 0$ these may be written in the form

$$
\sum_{i=0}^{n+2} a_{i} z^{i} \frac{\partial}{\partial z}+\sum_{j=0}^{n} b_{i} z^{i} \frac{\partial}{\partial w}
$$

with (1) ( $i$ even and $j$ odd) or ( $i$ odd and $j$ even), and

(2) $\left(a_{i}=-a_{n+2-i}\right.$ and $\left.b_{j}=b_{n-j}\right)$ or $\left(a_{i}=a_{n+2-i}\right.$ and $\left.b_{j}=-b_{n-j}\right)$.

Hence there are other nonsingular foliations in surfaces with $e=-1$, and since it is unbranchly covered by $\mathbf{C} \times E^{\prime}$, the theorem is proved.

Remark. It was noted by Etienne Ghys that the nonsingular foliations in elliptic ruled surfaces which are not Riccati are examples of minimal foliations which are not geodesible, since some leaves are parabolic, while most of them are hyperbolic [3]. Suwa [14] analysed those foliations in elliptic ruled surfaces that are branched fibrations.

\section{FAMilies of foliations}

In this section we describe the families of foliations by curves in ruled surfaces, when the complex structure of the ruled surface is fixed and when it is allowed to vary. We describe a large class of these families, and determine the Chern classes of the special families.

We have shown in [5] how Douady's parametrizations of quotient sheaves induces a natural complex analytic structure in the set of all foliations by curves $\mathscr{D}$ in the compact complex manifold $M$. The subsets $\mathscr{D}_{\alpha}$ formed by those foliations whose Chern class of the tangent bundle is $\alpha \in H^{2}(M, Z)$ is a union of connected components of $\mathscr{D}$, and it is compact. We also showed

Theorem 11 [5]. Let $M$ be a projective manifold and let $\alpha \in H^{2}(M, Z)$ be such that for any holomorphic line bundle on $M$ with Chern class $\alpha$ the vector space $H^{0}(M, \operatorname{Hom}(L, T M))$ is of constant dimension $r>0$, then $\mathscr{D}_{\alpha}$ has a natural structure of a $\mathbf{C} P^{r-1}$-bundle over a complex torus of dimension half the first Betti number of $M$.

Theorem 4.1. Let $\psi: S \rightarrow B$ be a ruled surface, and let $\alpha=a f+b h \in H^{2}(S, Z)$ lie in

$$
\alpha \in(2 c(\tau)+c(N)-\mathrm{Amp}) \cap(c(\tau)+2 c(N)-\mathrm{Amp}),
$$

then $\mathscr{D}_{\alpha}$ is a smooth manifold of dimension $2(a+2 g-2)(b-2)+3 g-3$, and it has a natural structure as a bundle of projective spaces over a torus of dimension $g$. 
Proof. Applying the functor $\operatorname{Hom}(L$,$) to (1.2), taking Euler-Poincare charac-$ teristics and using Lemma 1.3 we obtain

$$
\begin{aligned}
\chi\left(L^{*} \otimes T S\right) & =\chi\left(L^{*} \otimes \tau\right)+\chi\left(L^{*} \otimes N\right) \\
& =(-a+1-g)(-b+3)+(-a+3-3 g)(-b+1) \\
& =-b(-2 a+4-4 g)+(-4 a+6-6 g) \\
& =2(a-2+2 g)(b-2)+2 g-2 .
\end{aligned}
$$

Hypothesis (4.1) implies, by Lemma 1.3, that for $q>0 H^{q}\left(S, L^{*} \otimes \tau\right)=$ $H^{q}\left(S, L^{*} \otimes N\right)=0$. Hence $\operatorname{dim} H^{0}\left(S, L^{*} \otimes T S\right)=\chi\left(L^{*} \otimes T S\right)$ and we may apply the above stated theorem to finish the proof of this one.

Remark. The above theorem includes all families that contain foliations with isolated singularities, except the ruling and those satisfying for $e>0$

$$
\max (2-2 g-2 e, 4-4 g-e) \leq c(L) \cdot\left[B_{0}\right]^{*} \leq-e
$$

and $4-4 g \leq c(L) \cdot h \leq 2-2 g$ for $e \leq 0$. The foliations in these regions form special families. See Figure 3 . Note that the above also shows that every ruled surface admits a Riccati foliation, with at least $2 g-1$ vertical fibers as leaves.
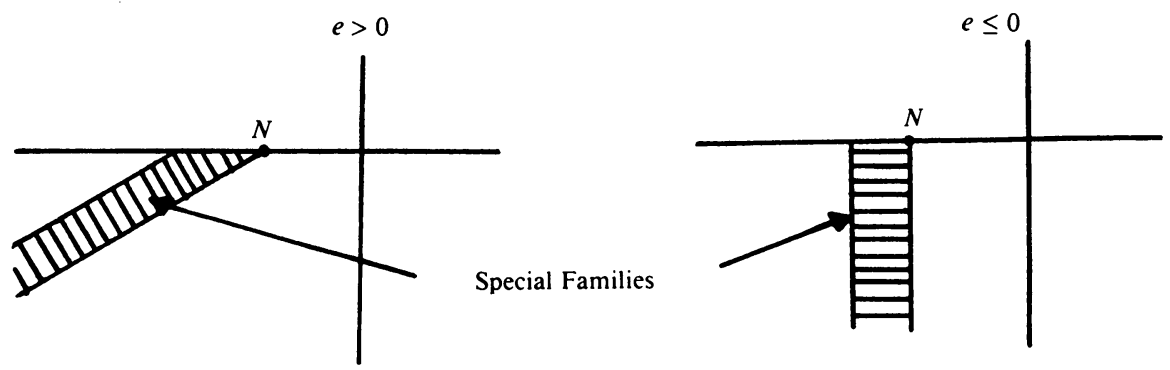

FIGURE 3

One can also consider families of foliations where the complex structure of the base manifold is varying and where two foliations are equivalent if there is a biholomorphism sending one onto the other one (see [6] where the existence of a versal space of deformations in this sense is shown to exist). We say that a ruled surface is stable if it is the projectivisation of a stable rank 2 vector bundle (in the sense of Mumford, see [11]).

Theorem 4.2. Let $\psi: S \rightarrow B$ be a stable ruled surface with $g>1$ and let $\alpha=a f+b h \in H^{2}(S, Z)$ be a cohomology class satisfying (4.1), then the versal space of deformations of $X: L \rightarrow T S$ with $c(L)=\alpha$ is a manifold of dimension $2(a+2 g-2)(b-2)+9 g-9$.

Proof. From the proof of Theorem 4.1 we obtain $\chi(T S)=6 g-6$. The long exact sequence of higher direct image sheaves of (1.2) imply that $R^{1} \psi_{*} \mathscr{O}_{S}(T S)=$ 0 , and hence $H^{2}\left(S, \mathscr{O}_{S}(T S)\right)=0$. The only automorphisms of a stable vector bundle are scalar multiples of the identity [11], so also $H^{0}\left(S, \mathscr{O}_{S}(T S)\right)=0$. 
From Kuranishi's Theorem [6] we have then that the versal space $\mathscr{V}_{S}$ is smooth of dimension $6 g-6$, and that it is locally universal. Using the Albanese mapping for example, we obtain a map $\mathscr{V}_{S} \rightarrow \mathscr{V}_{B}$ between the versal spaces of $S$ and $B$, each fiber is smooth and represents ruled surfaces over $B_{t}$. (Another way of seeing this is using Mumford's moduli of stable rank 2 vector bundles over $B_{t}$ [11] which has dimension $4 g-3$; and two vector bundles determine the same ruled surface if and only if one is a line bundle tensor the other one, so we obtain the $(3 g-3)$-dimensional smooth fibers.)

Repeating the proof of Theorem 11 in [5] applied to the relative Picard bundle over the family $\mathscr{V}_{S}$ we obtain as in Theorem 4.1 the versal space of foliations by curves of dimension $2(a+2 g-2)(b-2)+9 g-9$. This proves the theorem.

Applying Theorem 4.2 to Riccati foliations with $\alpha=-a f$ we obtain a family of dimension $4(a+2-2 g)+9 g-9=4 a+g-1$. This family may also be constructed using Proposition 2.7 since a Riemann surface of genus $g$ with $a+2-2 g$ punctures has moduli of dimension $3 g-3+(a+2-2 g)$. The fundamental group of such a punctured surface is free with $(2 g+a+2-2 g-1)=$ $a+1$ generators. The set of representations from the fundamental group to $\operatorname{PSL}(2, \mathrm{C})$ modulo conjugation is $3(a+1)-3=3 a$. In all we obtain by suspension a $4 a+g-1$ family. If the punctures are distinct, then the models which we complete are a countable number, so this process of suspension forms a dense open set of the set of all Riccati foliations.

Theorem 4.3. Let $C$ be a smooth irreducible curve in the ruled surface $\psi: S \rightarrow B$ with $[C]^{*}=c f+d h$, and let $\alpha=a f+b h \in H^{2}(S, Z)$ be a cohomology class satisfying

$$
\begin{gathered}
\alpha \in\left(2 c(\tau)+c(N)-[C]^{*}-\mathrm{Amp}\right) \cap\left(c(\tau)+2 c(N)-[C]^{*}-\mathrm{Amp}\right), \\
\alpha \cdot[C]^{*} \leq-2\left(c\left(\kappa_{S}\right)+[C]^{*}\right) \cdot[C]^{*} .
\end{gathered}
$$

Then the subvariety of the universal parameters $\mathscr{D}_{\alpha}$ formed by those foliations that are tangent to $C$ has dimension

$$
2 a b+(d-4) a+(c+4(g-1)) b+(-c d-c+(g-1)(d-5)) .
$$

Proof. The fundamental sequences of the subvariety $C \subset S$ are [6]:

$$
\begin{gathered}
0 \rightarrow \mathscr{O}_{S}(-C) \rightarrow \mathscr{O}_{S} \rightarrow \mathscr{O}_{C} \rightarrow 0, \\
\left.0 \rightarrow \boldsymbol{\theta}_{C} \rightarrow \boldsymbol{\theta}_{S}\right|_{C} \rightarrow \eta(S, C) \rightarrow 0 .
\end{gathered}
$$

$\eta(S, C)$ is the normal sheaf of $C$ in $S$. The long exact sequences of (4.4) tensored with $\Theta_{S} \otimes \mathscr{O}_{S}\left(L^{*}\right)$ and sequence (4.5) with $\mathscr{O}_{S}\left(L^{*}\right)$, where $L$ is a line bundle with Chern class $\alpha$ give the exact diagram

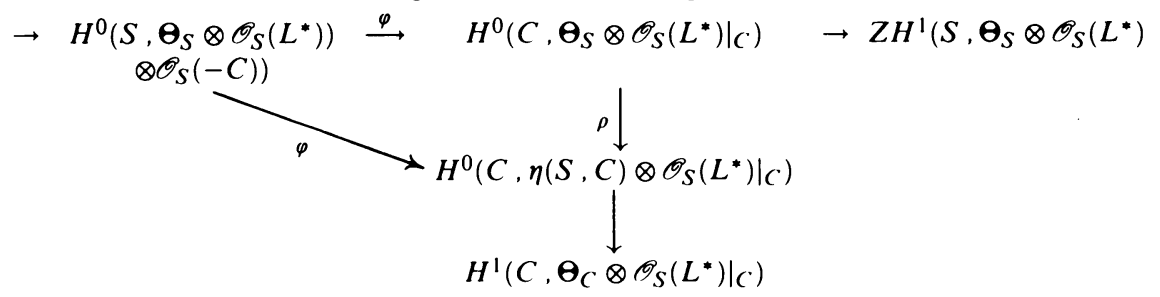


The set of maps $X: L \rightarrow T(S)$ such that their restriction to $C$ are tangent to $C$ is described by the kernel of $\bar{\varphi}$. Condition (4.2) is equivalent to having the sheaves $\mathscr{O}_{S}\left(\tau \otimes L^{*}\right) \otimes \mathscr{O}_{S}(-C)$ and $\mathscr{O}_{S}\left(N \otimes L^{*}\right)$ satisfy Lemma 1.3.3. Using (1.2) we obtain that for $q>0 H^{q}\left(S, \Theta_{C} \otimes \mathscr{O}_{S}\left(L^{*}\right) \otimes \mathscr{O}_{S}(-C)\right)=0$, so that $\varphi$ is surjective and by the computations in Theorem 4.1 for $q=0$ it has dimension

$$
\chi\left(c(\tau)-\alpha-[C]^{*}\right)+\chi\left(c(N)-\alpha-[C]^{*}\right)=2(c+a+2 g-2)(d+b-2)+2 g-2 \text {. }
$$

By the adjunction formula [7], the genus $g_{C}$ of $C$ is $\frac{1}{2}\left(c\left(\kappa_{S}\right)+[C]^{*}\right) \cdot[C]^{*}+1$; hence the Chern class of $\left.\Theta_{C} \otimes \mathscr{O}_{S}\left(L^{*}\right)\right|_{C}$ is $-\left(c\left(\kappa_{S}\right)+[C]^{*}+\alpha\right) \cdot[C]^{*}$. Condition (4.3) means that this Chern class is larger than $2 g-2$, so the first cohomology group vanishes, $\rho$ is surjective and by the Riemann-Roch formula, the space of global sections of $\left.\boldsymbol{\Theta}_{C} \otimes \mathscr{O}_{S}\left(L^{*}\right)\right|_{C}$ has dimension

$$
\text { (4.7) } 1-g_{C}-\left(c\left(\kappa_{S}\right)+[C]^{*}+\alpha\right) \cdot[C]^{*}=-(d a+c b+3 c d-3 c+(3 g-3) d) \text {. }
$$

The dimension of the kernel of $\bar{\varphi}$ is formed by adding (4.6) with (4.7). Adding to this number $g$ due to the variation of the complex structure in $L$ and subtracting a 1 due to the fact that two maps $X: L \rightarrow T S$ determine the same foliation if and only if one is a scalar multiple of the other, we obtained the stated formula.

Remark. By Theorem 4.1, the dimension of $\mathscr{D}_{\alpha}$ is asymptotically $2 a b$, so the codimension of the subvariety in Theorem 4.3 is asymptotically linear in $a$ and $b$. If $[C]^{*}$ belongs to $c\left(\kappa_{S}\right)+$ Amp, then by Lemma 1.3.3 and Bertini's Theorem [7] the set of curves that satisfy the hypothesis of the theorem has dimension $(c+1-g)(d+1)-1$. This shows that the families of foliations having at least one algebraic solution are arbitrarily large. The subset of Chern classes satisfying conditions (4.2) and (4.3) with $e>0$ and $C$ in Amp is of the form shown in Figure 4.

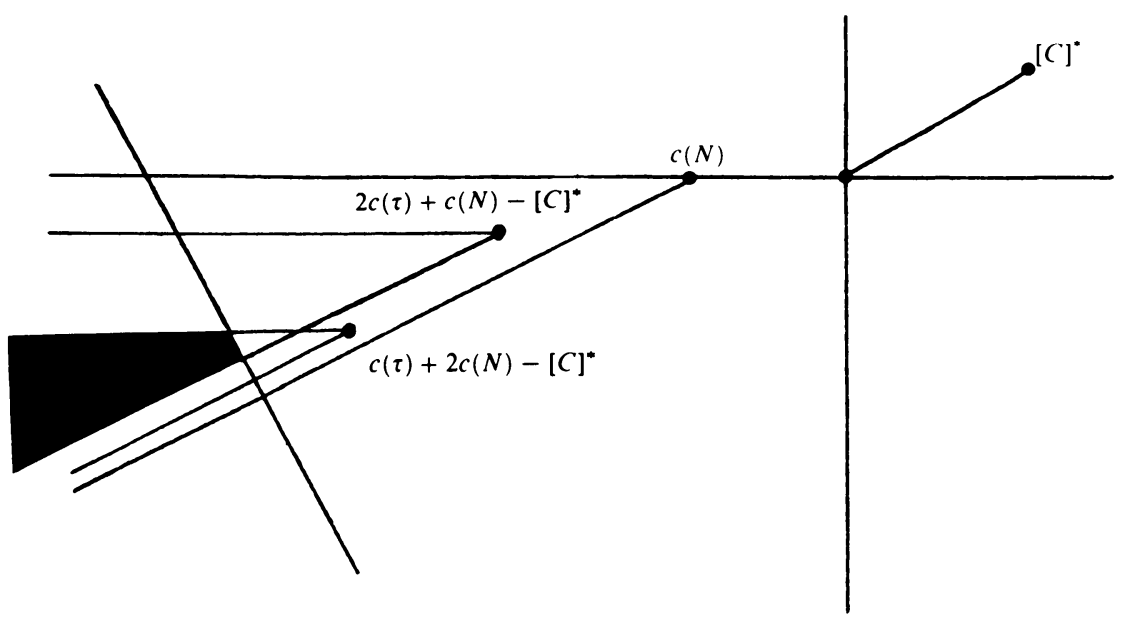

FiguRe 4 
We will now illustrate how the following general definition applies to ruled surfaces:

Definition 4.4. We say that a property $\mathscr{P}$ of foliations by curves is generic for projective manifolds if for every projective manifold $M$ and for every $\alpha, \beta \in H^{2}(M, Z)$, with $\beta$ ample, there is an $N$ such that for $n>N$ property $\mathscr{P}$ is satisfied for a dense $G_{\delta}$-set in $\{X: L \rightarrow T M / c(L)=\alpha-n \beta\}$.

The proof of Theorem 4.1 shows that it is a generic property for the moduli spaces $\mathscr{D}$ of foliations by curves to be smooth. We have shown in Theorem 6.7 of [6] that it is a generic property of foliations by curves to have singularities of multiplicity one. Due to the fact that there are an infinite number of components $\mathscr{D}_{\alpha}$, one should allow to exclude some special families. The notion of ample cone seems to be appropriate in this context, as it has been in the theory of special divisors on a Riemann surface [7]. Observe that conditions (4.1), (4.2) and (4.3) are satisfied in a set of the form $\gamma$-Amp, for a suitable $\gamma \in H^{2}(S, Z)$. Hence for ruled surfaces we have a stronger kind of genericity than the one needed for Definition 4.4, since there is a $G_{\delta}$-set in $\{X: L \rightarrow T M / c(L) \in$ $\gamma-\mathrm{Amp}\}$.

\section{RIGIDITY OF THE TRANSVERSE STRUCTURE}

In this section we will show, using the results of [6], that the only foliations with generic singularities that accept a change of the complex structure of the leaves keeping the transversal structure fixed are the rulings, Riccati foliations, Riccati-type foliations with respect to another fibre bundle structure and those foliations obtained from the complex projective plane $\mathbf{C} P^{2}$ by blowing up a nonsingular point. In the Riccati-type foliations, one keeps the representation fixed and one moves the location of the singular fibres; in the $\mathbf{C} P^{2}$ case, we obtain a one-dimensional family by moving the blown-up point in $\mathbf{C} P^{2}$ within the same leaf. Using [6], the proof will consist in finding those foliations $X: L \rightarrow T S$ such that $H^{1}\left(S, \mathscr{O}_{S}(L)\right) \neq 0$. We will first analyse surfaces with irregularity $g>1$, and afterwards we will deal with the special cases $g=0,1$.

Proposition 5.1. Let $X: L \rightarrow T S$ be a foliation by curves with isolated zeros in the ruled surface $\psi: S \rightarrow B$, then

(1) If $X$ defines the ruling, then

$$
\operatorname{dim} H^{1}\left(S, \mathscr{O}_{S}(L)\right)=3 g-3+\operatorname{dim} H^{0}\left(S, \mathscr{O}_{S}(L)\right) .
$$

(2) If $X$ is a Riccati foliation with $c(L)=a f$, then $\operatorname{dim} H^{1}\left(S, \mathscr{O}_{S}(L)\right)=$ $3 g-3+(2-2 g-a)$.

(3) In all other cases with $g>1$ we have $H^{1}\left(S, \mathscr{O}_{S}(L)\right)=0$.

Proof. In case $1, c(L)=2 h$, so that Lemma 1.3 gives $\chi(L)=3-3 g$. Since $R^{1} \psi_{*} \mathscr{O}_{S}(L)=0$, we have that $H^{2}\left(S, \mathscr{O}_{S}(L)\right)=0$. This gives part 1 . 
For case 2, let $L=\psi^{*}\left(L_{1}\right), X: L \rightarrow T S$ the map describing the foliation and $\bar{X}: L \rightarrow N=\psi^{*} T B$ the map of (2.1). Since $R^{0} \psi_{*} \mathscr{O}_{S}(L)=L_{1}$ and $R^{1} \psi_{*} \mathscr{O}_{S}(L)=0$ we have that $H^{1}\left(S, \mathscr{O}_{S}(L)\right)=H^{1}\left(B, \mathscr{O}_{B}\left(L_{1}\right)\right)$. The map $\bar{X}$ is a global section of $H^{0}\left(S, \mathscr{O}_{S}\left(L^{*} \otimes N\right)\right)$, but by a similar argument as for $L$ this group is isomorphic to $H^{0}\left(B, \mathscr{O}_{B}\left(L_{1}^{*} \otimes T B\right)\right)$. Denote by $\bar{x}$ the section corresponding to $X$. From the proof of Proposition 2.7 we may interpret $\bar{x}=0$ as representing the fibers of $\psi$ which are tangent to the foliation. If we call this set $p_{1}+\cdots+p_{2-2 g-a}$ then $\mathscr{O}_{B}\left(L_{1}\right)$ is isomorphic to $\boldsymbol{\Theta}_{B}\left(p_{1}+\cdots+p_{2-2 g-a}\right)$, so that canonically $H^{1}\left(S, \mathscr{O}_{S}(L)\right) \simeq H^{1}\left(B, \Theta_{B}\left(p_{1}+\cdots+p_{2-2 g-a}\right)\right)$. This last group has dimension $3 g-3+(2-2 g-a)$, and its classical interpretation is the infinitesimal deformations of the punctured Riemann surface $B-\left\{p_{1}, \ldots, p_{2-2 g-a}\right\}$.

Part 3 follows from part 4 of Lemma 1.3. This finishes the proof.

Remark. Since the ruling is topologically rigid by Reeb's stability theorem, part 1 is detecting the different bundle structures that can be put on $S$ with the complex structure of $B$ fixed. If $S$ is stable, the dimension is $3 g-3$.

Proposition 5.2. Let $X: L \rightarrow T S$ be a foliation with isolated singularities in the elliptic ruled surface $\psi: S \rightarrow B$. If $H^{1}\left(S, \mathscr{O}_{S}(L)\right) \neq 0$, then $X$ is a Riccati foliation or nonsingular.

Proof. By Proposition 2.2 we know that if $X$ is not the ruling, then $c(L) \in$ - $\overline{\mathrm{Amp}}$. Part 4 of Lemma 1.3 assures that $H^{1}\left(S, \mathscr{O}_{S}(L)\right)=0$ if $c(L) \in-$ Amp ; hence we only have to analyse those bundles with Chern class in $-(\overline{\mathrm{Amp}}-\mathrm{Amp})$; i.e. the two boundary lines in Figure 2 with $N=0$. The horizontal line corresponds to Riccati foliations, and it is easy to compute that $H^{1}\left(S, \mathscr{O}_{S}(L)\right) \neq$ 0 for this case. We have to analyse the behaviour on the nonhorizontal axis. If the invariant $e \leq 0$, we have shown in $\S 3$ that these foliations are nonsingular and of Riccati-type. Using the representation of them as quotients of Example 3.1 and using equivariant cohomology, one sees that $H^{1}\left(S, \mathscr{O}_{S}(L)\right) \neq 0$ for most of them.

Assume then that $e>0$. We may represent $S$ as $S=\operatorname{Proj}\left(\mathscr{O}_{C} \oplus \mathscr{L}\right)$, where $\mathscr{L}$ is an invertible sheaf on $B$ of Chern class $-e$ [8, p. 377]. Let $\mathscr{H}$ be the sheaf of relative hyperplanes on $S$, whose nontrivial sections define the minimal self-intersection $\psi$-section. Hence $c(\mathscr{H})=-\frac{1}{2} e f+h$ and $\mathscr{H} \otimes \psi^{*} \mathscr{L}^{*}$ has Chern class $\frac{1}{2} e f+h$. We are interested in computing

$$
H^{1}\left(S,\left(\mathscr{H}^{*} \otimes \psi^{*} \mathscr{L}\right)^{n} \otimes \psi^{*} \mathscr{L}_{0}\right)=H^{1}\left(S, \mathscr{L}^{n-2} \otimes \psi^{*} \mathscr{L}^{-n} \otimes \psi^{*} \mathscr{L}_{1}\right)^{*}
$$

with $n>0$; where $\mathscr{L}_{0}$ and $\mathscr{L}_{1}$ are invertible sheaves on $B$ with Chern class 0 , and for the last equality we have used Serre duality and $c\left(\Omega_{S}^{2}\right)=-2 h$.

For $n>0$ we have $R^{1} \psi_{*}\left(\mathscr{H}^{n-2}\right) \otimes\left(\mathscr{L}^{-n} \otimes \mathscr{L}_{1}\right)=0$, and for $n>1$ we have

$$
\begin{aligned}
\psi_{*}\left(\mathscr{L}^{n-2}\right) \otimes \mathscr{L}^{-n} \otimes \mathscr{L}_{1} & =\operatorname{Sym}^{n-2}\left(\mathscr{O}_{B} \oplus \mathscr{L}\right) \otimes\left(\mathscr{L}^{-n} \otimes \mathscr{L}_{1}\right) \\
& =\left(\mathscr{O}_{B} \oplus \mathscr{L} \oplus \cdots \oplus \mathscr{L}^{n-2}\right) \otimes\left(\mathscr{L}^{-n} \otimes \mathscr{L}_{1}\right)
\end{aligned}
$$


while for $n=1$ it is 0 . The first cohomology group of (5.2) is 0 , since all the summands have positive Chern class. Hence by the degenerate spectral sequence of higher direct image sheaves we obtain that $H^{1}\left(S, \mathscr{O}_{S}(L)\right)=0$ if $c(L)=-n\left(\frac{1}{2} e f+h\right)$ and $n>0$. This finishes the proof of the proposition.

We analyse the case $g=0$. Let $\psi: S_{e}=\operatorname{Proj}(\mathscr{O}(0) \oplus \mathscr{O}(e)) \rightarrow P^{1}$ be the ruled surface over $P^{1}$ obtained by projectivising $\mathscr{O}(0) \oplus \mathscr{O}(e), e \geq 0$, where $\mathscr{O}(j)$ is the invertible sheaf on $P^{1}$ with Chern class $j$. We shall prove

Proposition 5.3. Let $X: L \rightarrow T S_{e}$ be a foliation with isolated zeros in the rational ruled surface $\psi: S_{e} \rightarrow P^{1}$, different from the ruling or a Riccati foliation; then $H^{1}\left(S_{e}, \mathscr{O}(L)\right)=0$ unless

(1) $e=0$ and $X$ is a Riccati foliation with respect to the other projection, $\psi^{\prime}: S_{0}=P^{1} \times P^{1} \rightarrow P^{1}$.

(2) $e=1$ and $c(L)=f-n\left(\frac{1}{2} f+h\right)$. In this case $H^{1}\left(S_{1}, \mathscr{O}(L)\right)$ has dimension one.

Proof. We will begin by proving sharper vanishing estimates than the ones contained in Lemma 1.3. By [8, p. 162], we have that for $n \geq 0$

$$
\psi_{*} \mathscr{H}^{n}=\bigoplus_{j=0}^{n} \mathscr{O}(j e) \quad \text { and } \quad R^{q} \psi_{*} \mathscr{H}^{n}=0 \text { for } q>0 ;
$$

where $\mathscr{H}$ is the sheaf of relative hyperplane sections on $\operatorname{Proj}(\mathscr{O}(0) \oplus \mathscr{O}(e))$. We also have that $R^{q} \psi_{*} \mathscr{H}^{*}=0$ for $q \geq 0$, since $H^{q}\left(P^{1}, \mathscr{O}(-1)\right)=0$ for $q \geq 0$. Hence the spectral sequence of $\psi_{*}\left(\mathscr{L}^{n} \otimes \psi^{*} \mathscr{O}(m)\right)$ degenerates for $n \geq-1$; and we obtain for $n \geq 0$ and $m \geq-1$ :

$$
\begin{aligned}
& H^{0}\left(S_{e}, \mathscr{H}^{n} \otimes \psi^{*} \mathscr{O}(m)\right)=\bigoplus_{l=0}^{n} H^{0}\left(P^{1}, \mathscr{O}(l e+m)\right), \\
& H^{q}\left(S_{e}, \mathscr{H}^{n} \otimes \psi^{*} \mathscr{O}(m)\right)=0 \quad \text { for } q>0
\end{aligned}
$$

and

$$
H^{q}\left(S_{e}, \mathscr{H}^{*} \otimes \psi^{*} \mathscr{O}(m)\right)=0 \text { for } q>0, m \in Z .
$$

The projectivisation of $\mathscr{O}(e)$ in $S_{e}=\operatorname{Proj}(\mathscr{O} \oplus \mathscr{O}(e))$ gives rise to the minimal self-intersection $\psi$-section $B_{0} . B_{0}$ has self-intersection number $-e$, since its normal sheaf is isomorphic to the restriction to $B_{0}$ of $\psi^{*} \mathscr{O}(-e)$. Hence $B_{0}$ is the vanishing set of a section of $\mathscr{H} \otimes \mathscr{O}(-e)$. We then deduce that the Chern class of $\mathscr{H}$ is $\left[B_{0}\right]^{*}+e f=\frac{1}{2} e f+h$.

Formula (5.3) means that for any line bundle $L$ on $S_{e}$ with Chern class of the form $m f+n\left(\frac{1}{2} e f+h\right)$, with $m \geq-1$ and $n \geq 0$, we have $H^{q}\left(S_{e}, \mathscr{O}(L)\right)=0$ for $q>0$. By Serre duality, we also obtain that

$$
\begin{aligned}
H^{q}\left(S_{e}, \mathscr{O}(L)\right) & =0 \text { for } q<2 \text { if } c(L) \\
& =-2 f-2 h+m f+n\left(\frac{1}{2} e f+h\right), \quad m \leq 1, n \leq 0 .
\end{aligned}
$$


Also from (5.4) we have

$$
H^{q}\left(S_{e}, \mathscr{H}^{*} \otimes \psi^{*} \mathscr{O}(m)\right)=0 \text { for } q \geq 0 \text { and } m \in Z .
$$

Let $X: L \rightarrow T S_{e}$ be as in the statement of the proposition. Using Proposition 2.2 and Lemma 1.2, we have that the Chern class of $L$ is of the form $c(L)=2 f-m^{\prime} f-n^{\prime}\left(\frac{1}{2} e f+h\right)$, with $m^{\prime} \geq 0$ and $n^{\prime}>0$. If $n^{\prime}=1$, then $H^{1}\left(S_{e}, \mathscr{O}(L)\right)=0$ by (5.6), as well if $e>2$ by (5.5). Hence we have proved the proposition for $e>2$, and we will now analyse the exceptional case $e=0,1,2$.

For $e=0$, applying (5.5) to both rulings, and (5.6) to $-f-h$, we have that $H^{1}\left(S_{e}, \mathscr{O}(L)\right)=0$, except if $X$ is a Riccati foliation or the rulings.

For $e=1$, the preceding argument works except for $2 f-n\left(\frac{1}{2} f+h\right)$ and $f-n\left(\frac{1}{2} f+h\right)$, with $n \geq 2$. We will begin by showing that the first case does not appear with isolated singularities. To see this, recall that

$$
\Phi: \mathbf{C}^{2} \rightarrow \mathbf{C}^{2}, \quad \Phi\left(z_{1}, z_{2}\right)=\left(z_{1}^{-1}, z_{1}^{e} z_{2}\right)=\left(w_{1}, w_{2}\right)
$$

is a coordinate description of $S_{e}$; where $B_{0}$ is defined by $z_{2}=0$. The curve $B_{1}$ defined by $z_{2}=\infty$ has fundamental class $\left[B_{1}\right]^{*}=\frac{1}{2} f+h$ and the fiber $F$ defined by $w_{1}=0$ has fundamental class $[F]^{*}=f$.

The morphism $X: L \rightarrow T S_{1}$ may be expressed by a polynomial vector field in $\left(z_{1}, z_{2}\right)$ having a pole of order at most $n$ on $B_{1}$ and a zero of order at least 2 on $F$. The vector field

$$
X\left(z_{1}, z_{2}\right)=\sum_{j=0}^{n} a_{i j} z_{1}^{i} z_{2}^{j} \frac{\partial}{\partial z_{1}}+\sum_{j=0}^{n+2} b_{i j} z_{1}^{i} z_{2}^{j} \frac{\partial}{\partial z_{2}}
$$

has a pole of order at most $n$ on $B_{1}$. We claim that in order for $X$ to have a zero of order 2 on $F$ it has to vanish also along $B_{1}$. To see this, we represent $X\left(z_{1}, 0\right)$ in the $w$-coordinates

$$
D \Phi(X)=\left(\begin{array}{cc}
-w_{1}^{2} & 0 \\
w_{1} w_{2} & w_{1}^{-1}
\end{array}\right)\left(\begin{array}{c}
\sum a_{i j} w_{1}^{j-i} w_{2}^{j} \\
\sum b_{i j} w_{1}^{j-i} w_{2}^{j}
\end{array}\right) .
$$

In order that the first coordinate in (5.9) has a zero of order 2 when $w_{2}=0$ we need to have $a_{i 0}=0$ for $i>0$. But

$$
D \Phi\left(a_{00} \frac{\partial}{\partial z_{1}}\right)=a_{00}\left(-w_{1}^{2} \frac{\partial}{\partial w_{1}}+w_{1} w_{2} \frac{\partial}{\partial w_{2}}\right)
$$

and the term $w_{1} w_{2}\left(\partial / \partial w_{2}\right)$ cannot be cancelled with any term of the form $b_{i j} w_{1}^{j-i-1} w_{2}^{j}$, since $i \geq 0$. Hence $a_{i 0}=0$ for $i>0$. Also we need to have $b_{i 0}=0$ if $X$ is to have a zero of order 2 on $F$. Hence $X$ vanishes on $B_{0}$, which is contrary to the hypothesis. We have then showed that there is no foliation with isolated singularities and Chern class of the form $2 f-n\left(\frac{1}{2} f+h\right)$.

Now, assume that the Chern class of $L$ is of the form $f-n\left(\frac{1}{2} f+h\right)$, with $n \geq 2$. First observe that

$$
H^{1}\left(S_{1}, \mathscr{O}(L)\right) \cong H^{1}\left(S_{1}, \mathscr{O}\left(L^{*}\right) \otimes \Omega^{2}\right)
$$


where the Chern class of the sheaf in the right term is $-2 f+(n-2)\left(\frac{1}{2} f+h\right)$. Hence $R^{1} \psi_{*}\left(\mathscr{O}\left(L^{*}\right) \otimes \Omega^{2}\right)=0$ and $\psi_{*}\left(\mathscr{O}\left(L^{*}\right) \otimes \Omega^{2}\right)=\bigoplus_{j=0}^{n-2} \mathscr{O}(j-2)$. Hence the spectral sequence of higher direct image sheaves degenerates and

$$
H^{1}\left(S_{1}, \mathscr{O}\left(L^{*}\right) \otimes \Omega^{2}\right) \cong \mathrm{C} .
$$

To see that there are foliations in $S_{1}$ with this Chern class, recall that $S_{1}$ is obtained from the complex projective plane $\mathbf{C} P^{2}$ by blowing up a point $p_{0}$, $\sigma: S_{1} \rightarrow P^{2}$, where the ruling comes from all the lines in $\mathbf{C} P^{2}$ through $p_{0}$. Let $\mathscr{F}$ be a foliation in $\mathbf{C} P^{2}$, nonsingular on $p_{0}$. Let $Y$ be a rational vector field on $S_{1}$, tangent to $\mathscr{F}$ and having its poles on a line $\mathscr{L}$ with multiplicity $n-1$, which does not contain $p_{0}$ and with isolated zeros in $P^{2}-\mathscr{L} \cdot \sigma^{*} Y$ is a rational vector field on $S_{1}$ having a pole of order $n-1$ on $\sigma^{-1}(\mathscr{L})$, $\left[\sigma^{-1}(\mathscr{L})\right]^{*}=\frac{1}{2} f+h$, having a pole of order 1 on the exceptional divisor, $\left[\sigma^{-1}\left(p_{0}\right)\right]^{*}=-\frac{1}{2} f+h$, and with isolated zeros. Cancelling the poles of $\sigma^{*}(Y)$, we obtain a bundle map $X: L \rightarrow T S_{1}$ where the Chern class of $L$ is

$$
-(n-1)\left(\frac{1}{2} f+h\right)-\left(-\frac{1}{2} f+h\right)=-n\left(\frac{1}{2} f+h\right)+f .
$$

To finish the proof of the proposition, note that the argument at the beginning of the proof works for $e=2$ except for Chern classes of the form $2 f-$ $n(f+h)$. Using the coordinate description (5.7) of $S_{2}$, and carrying out a similar argument as the one presented for $e=1$ and $c(L)=2 f-n\left(\frac{1}{2} f+h\right)$, we conclude that any such bundle map $X: L \rightarrow T S_{2}$ vanishes on $B_{0}$. This proves the proposition.

Remark. Using the results of [6], we obtain that for a dense open subset of the subvariety of $\mathscr{D}_{\alpha-n \beta}$ defined in Theorem 4.3, with $n$ sufficiently large and ample $\beta$, the topological class of the foliation determines the holomorphic class.

\section{REFERENCES}

1. M. F. Atiyah, Vector bundles over an elliptic curve, Proc. London Math. Soc. 27 (1957), $414-$ 452.

2. D. Birkoff, The generalized Riemann problem for linear differential equations, Proc. Amer. Acad. Arts Sci. 49 (1913), 521-568.

3. G. Cairns and E. Ghys, Totally geodesic foliations on four manifolds, J. Differential Geometry 23 (1986), 241-254.

4. S. S. Chern, Meromorphic vector fields and characteristic numbers, Scripta Math. 29 (1973), 243-251.

5. X. Gómez-Mont, Universal families of foliations by curves, Proc. Conf. Dyn. Syst., Dijon, 1985 (D. Cerveau, R. Moussou, eds.), Astérisque 150-151 (1987), 109-129.

6. _. The transverse dynamics of a holomorphic flow, Ann. of Math. 127 (1988), 49-92.

7. Ph. Griffiths and J. Harris, Principles of algebraic geometry, Wiley Interscience, 1978.

8. R. Hartshorne, Algebraic geometry, Springer-Verlag, Berlin and New York, 1977.

9. F. Hirzebruch, Topological methods in algebraic geometry, Springer-Verlag, Berlin and New York, 1966. 
10. J. Martinet and J. F. Ramis, Problèmes de modules pour des équations différentielles non linéaires du prémier ordre, Publ. Math. Inst. Hautes Études Sci. 55 (1982), 63-164.

11. P. Newstead, Introduction to moduli problems and orbit spaces, Tata Inst., Bombay, 1978.

12. P. Painlevé, Leçons sur la théorie analytique des équations differentielles, professées à Stockholm, 1895, Oeuvres, CNRS, 1972.

13. D. Sundararaman, Compact Hausdorff transversely holomorphic foliations, Lecture Notes in Math., vol. 950, Springer-Verlag, Berlin and New York, 1982, pp. 360-374.

14. T. Suwa, Ruled surfaces of genus one, J. Math. Soc. Japan 21 (1969), 291-311.

Instituto de Matemáticas, U.N.A.M., Ciudad Universitaria, 04510, México, D.F. Mexico 